\title{
Usage characteristics and adverse event rates of the direct puncture and pull techniques for percutaneous endoscopic gastrostomy in patients with malignant tumors of the upper aerodigestive tract
}

\section{다 (우우}

\section{Authors}

Niels Teich ${ }^{1,2,3}$, Lars Selig ${ }^{4}$, Susanne Liese ${ }^{5}$, Franziska Schiefke ${ }^{6,7}$, Alexander Hemprich ${ }^{6}$, Joachim Mössner ${ }^{4}$, Ingolf Schiefke ${ }^{1,8}$

Institutions

1 Klinikum St. Georg, Klinik für Gastroenterologie und Hepatologie, Leipzig, Germany

2 Internistische Gemeinschaftspraxis für Verdauungs- und Stoffwechselkrankheiten, Leipzig, Germany

3 Medizinische Fakultät der Friedrich-Schiller-Universität Jena, Jena

4 Universität Leipzig, Klinik und Poliklinik für Gastroenterologie und Rheumatologie, Leipzig, Germany

5 Universität Rostock, Klinik und Poliklinik für Mund-, Kiefer- und Plastische Gesichtschirurgie, Rostock, Germany

6 Universität Leipzig, Klinik für Mund-, Kiefer- und Plastische Gesichtschirurgie, Leipzig, Germany

7 Gemeinschaftspraxis für Mund-, Kiefer- und Gesichtschirurgie am Johannisplatz, Leipzig, Germany

8 Gastroenterologie und Hepatologie am Johannisplatz, Leipzig, Germany

submitted 14.5.2017

accepted after revision 12.9.2017

Bibliography

DOI https://doi.org/10.1055/s-0043-121879 |

Endoscopy International Open 2018; 06: E29-E35

(c) Georg Thieme Verlag KG Stuttgart · New York

ISSN 2364-3722

Corresponding author

Niels Teich, MD, Internistische Gemeinschaftspraxis für Verdauungs- und Stoffwechselkrankheiten, Nordstr. 21, D-04105 Leipzig, Germany

Fax: +004934160036560

teich@igvs.de

\section{ABSTRACT}

Background and study aims Patients with malignant tumors of the upper gastrointestinal tract are at risk of weight loss. Early supportive nutrition therapy is therefore recommended and usually requires placement of a percutaneous endoscopic gastrostomy (PEG). The aim of this study was to compare adverse events and usage characteristics of the direct puncture technique with those of the traditional pull technique when used in patients with endoscopically passable tumors. The primary endpoint was the rate of inflammatory adverse events (AEs) at the gastrostomy fistula. The secondary endpoint was the long-term rate of puncture-site metastases.

Patients and methods One hundred twenty patients (median age 56; IQR 36, 86 years) were randomized and treated per protocol in this prospective open randomized single-center study. Follow-ups were conducted on the third and seventh post-interventional days, after 1, 3 and 6 months and the last follow-up 5 years after intervention.

Results Within the short-term follow-up period of 6 months after PEG placement, AEs were noted in 47 patients (39.2\%). These included 22 inflammations and 16 device dislocations and were mainly found in the puncture group (33 vs. 14 in the pull group) with a significantly increased incidence in the first month after PEG insertion $(P=0.001)$. Evaluation of the 5-year data did not reveal any significant differences. The gastrostomy tube was used in 101 patients (84.2\%) (range 18 days to 5 years).

Conclusions Our results favor the pull technique for patients with endoscopically passable tumors of the upper gastrointestinal tract due to less short-term adverse events. Both systems contributed equally to secure long-term use. 


\section{Introduction}

Due to local and systemic tumor effects and as a consequence of tumor-specific therapy, $30 \%$ to $80 \%$ of patients with tumors lose weight. Patients with tumors of the upper gastrointestinal tract are particularly at risk of weight loss due to impaired chewing and swallowing. Consequently, malnutrition correlates with impaired prognosis [1]. ESPEN Guidelines on Enteral Nutrition suggest that supportive nutrition therapy should be started only if malnutrition is already present $(\mathrm{BMI}<18.5)$ or if the patient is unable to consume less than $60 \%$ of the required daily energy intake over a period of more than 7 to 10 days [2]. If low nutrient supply is expected to last longer than 2 to 3 weeks, placement of a percutaneous endoscopic gastrostomy (PEG) should be favored over nasoenteric systems [2]. One advantage of this method is the long viability and turnaround time largely due to the safe fixation of the tube by intragastric and extragastric retention plates. The most common PEG technique is the pull technique, which was first described in 1980 by Gauderer et al. After conventional esophagogastroduodenoscopy and percutanous puncture of the stomach, a feeding tube with an intragastrically placed retention plate passes the upper digestive tract [3]. As a modification, with the introducer technique, the tube is placed via Seldinger technique using a guidewire.

A unique adverse event (AE) of PEG insertion in patients with head and neck cancer is tumor seeding and formation of stromal metastases on the abdominal wall and viscera, especially the stomach. That was first described by Preyer and Thul in 1989 [4]. In a review of all 44 reported cases, Cappell concluded that strong risk factors for stomal metastases included primary pharynx-esophageal cancer, squamous cell histology, less differentiated tumor, large size and advanced cancer stage, PEG placement by the pull technique, untreated primary cancer with local recurrence and at a time $>3$ months after PEG insertion [5]. Several authors have recommended use of direct endoscopic introducer technique (Russell's technique) or insertion under radiological guidance to help avoid this dreaded $\mathrm{AE}$ [5-7].

Until now there have been only a few comparative studies related to PEG placement by means of the pull technique or the direct puncture technique. Most of them were retrospective and did not reveal consistent results [8-14]. Almost all studies enrolled in a recent meta-analysis were observational studies without randomization of insertion methods, and none of the groups were matched exactly [15]. Although gastrostomy has been widely used, there is a lack of randomized trials on this issue.

In accordance with the state of evidence at the time of initiation of our study, we hypothesised that the puncture technique would decrease the frequency of inflammatory AEs and rate of formation of metastases at the gastrostomy fistula in patients with tumors in the upper gastrointestinal tract. Therefore, we investigated in an open prospective trial whether application of the direct puncture technique (Freka-PEXACT-System) is superior to the pull technique. Moreover, we aimed to compare the usage characteristics in the long term.

\section{Patients and methods}

The primary endpoint was the rate of inflammatory AEs in the area of the gastrostomy fistula. The working hypothesis postulated that use of the Freka-PEXACT-System during placement of a PEG in patients with epithelial tumors of the upper gastrointestinal tract or the larynx would reduce the rate of inflammatory AEs in the gastrostomy area as compared to PEG placement using the pull technique as the PEG device would not be in direct contact with the microbiome of the upper gastrointestinal tract. Under the assumption that bypassing the upper gastrointestinal tract via the Freka-PEXACT-System would reduce the frequency of metastases, the frequency of metastases in the stoma area was defined as the secondary endpoint of the study.

The study was conducted as a monocenter, open, randomized, prospective trial at Leipzig University. Recruitment took place over 18 months and started in January 2007. Inclusion and exclusion criteria are listed in $\mathbf{B}$ Box. Assignment into the pull group (traditional PEG placement using the pull technique) or into the puncture group (PEG placement using the FrekaPEXACT-System) was continuous and changed on a monthly base. This resulted in well balanced patient assignments to both groups ( $\vee$ Table 1 ). The study participants were examined over a median period of 53 months (minimum/maximum 1 month/101 months, interquartile range 12.75; 96 months) after PEG placement. The study protocol was voted on favorably by the Ethics Committee of the Medical Faculty of the University of Leipzig.

\section{INCLUSION AND EXCLUSION CRITERIA}

\section{Inclusion criteria}

- Evidence of a histologically confirmed malignant epithelial tumor of the upper gastrointestinal tract or larynx

- Tumor passable by standard gastroscope (external diameter $\geq 8.7 \mathrm{~mm}$ )

- Evidence of a safe diaphanoscopy

- Age $\geq 18$ years

- Written consent from patient or his/her legal representative for the placement of a PEG and participation in this study after full procedural disclosure (24 hours before the examination)
Exclusion criteria
- Gastric varices
- Coagulopathies
- Peritonitis
- Gastric ulcer in the front wall
- Ascites
- Status post-resection stomach surgery
- Upper gastrointestinal tract stenosis including
- patients with neurological dysphagia (alone or concomitant)
- Pregnancy 
- Table 1 Patient characteristics showing no significant differences between groups.

\begin{tabular}{|l|l|l|}
\hline \multirow{2}{*}{ Characteristics } & \multicolumn{2}{|c}{ Patients } \\
\cline { 2 - 3 } & Pull technique & Direct puncture \\
\hline Age & median (IQR) & median (IQR) \\
\hline BMI & $71(59-69)$ & $67(60-75)$ \\
\hline & $23(20-26)$ & $23(21-27)$ \\
\hline Male & $n(\%)$ & $n(\%)$ \\
\hline Indication & $51(86)$ & $55(84)$ \\
\hline - Prior chemotherapy & $16(27)$ & $12(18)$ \\
\hline - Insufficient oral intake & $39(66)$ & $51(78,5)$ \\
\hline - Prior operation & $3(5)$ & $1(1)$ \\
\hline - No oral intake at all & $1(2)$ & $1(2)$ \\
\hline Location & & $10(16)$ \\
\hline - Oral cavity & $16(27)$ & $23(35)$ \\
\hline - Pharynx & $31(52)$ & $31(47)$ \\
\hline - Larynx & $10(17)$ & $1(3)$ \\
\hline - Esophagus & & \\
\hline BMI, body mass index & & \\
\hline
\end{tabular}

After patients' approval via written consent for participation in the study and diagnostic esophagogastroduodenoscopy, PEG placement was performed under sterile conditions and $2 \mathrm{~g}$ ceftriaxon were administered intravenously for antibiotic prophylaxis for 30 to 60 minutes during the pre-interventional period. A skin shave was done if appropriate and the patients were placed in a supine position. We did not carry disinfect the oral cavity or decontaminate the gut with antibiotics. Propofol sedation was used in all cases. A 1-cm skin incision prior to insertion of the PEG was done after transillumination in all patients. For the pull technique, we used Freka PEG 15 Charier (Fresenius Kabi AG, Bad Homburg, Germany) in accordance with the manufacturer's recommendations. Fixation of the PEG tube was reinforced by means of an interior and exterior retention plate without sutures.

For PEG placement using the direct puncture technique, we used the Freka-PEXACT-System (Fresenius Kabi AG, Bad Homburg, Germany). In brief, this required prior safe fixation of the stomach front wall to the abdominal wall. For that purpose, 2 gastropexies were performed and placed laterally to the puncture site using a gastropexy device in accordance with the manufacturer's instructions. Following the stab incision, a trocar and, subsequently, a pull-away sleeve were inserted over which a balloon probe was placed and fixed by filling the balloon with $5 \mathrm{~mL}$ of sterile water. Four weeks later, this was replaced with a permanent Freka Button 15 Charier (Fresenius Kabi AG, Bad Homburg, Germany, filled with $5 \mathrm{~mL}$ of sterile saline). For that purpose, the gastropexy stitches were removed and the feeding tube was exchanged percutaneously using a guidewire.

Follow-up for observation of early AEs was conducted on the Days 3, 7 and 21 post-intervention. To include possible late AEs, further follow-up was carried out after 1, 3 and 6 months and a last follow-up 5 years after intervention.

The occurrence of inflammatory AEs at the PEG placement site was determined both clinically and anatomically. The occurrence of local signs of inflammation was also recorded.

Subjective patient satisfaction with the feeding tube was estimated using a visual analogue scale (VAS) on which patients marked the level of their satisfaction along a $100-\mathrm{mm}$ straight line.

Computer-facilitated scoring and graphic representation of the collected data were performed using Microsoft Office Excel 2007 and SPSS 15.0 for Microsoft Windows. The following techniques were used for statistical evaluation: Mann-Whitney $U$ test, chi-squared test, Fisher's exact test, Wilcoxon test, Kaplan-Meier survival analysis, logrank test, as appropriate.

This trial was not registered through any of the registries approved by the International Committee of Medical Journal Editors as this was not mandatory at the time of initiation of our study in 2007.

\section{Results}

\section{Patient characteristics}

One hundred thirty-eight patients were screened for this study and 120 of them were randomized ( $\triangleright$ Fig. 1 ). The median age of the per protocol cohort was 64 years (IQR: 59; 74 years), $85 \%$ of study participants were men. The most frequent localization of the malignant disease was the pharynx (50\%); followed by the oral cavity $(31.5 \%)$, the larynx (16.1\%) and the esophagus (2.4\%). The median body mass index (BMI) was 23 (IQR: 21, 27). The median initial Karnofsky Index was $80 \%$ (40-90\%). There was no significant difference in BMI and Karnofsky indices between the groups. 58 patients (48.3\%) were assigned to the pull group, 62 patients ( $51.7 \%$ ) were assigned to the puncture group ( $\triangleright$ Table 1$)$.

\section{PEG procedure time}

Procedure time for successful placement of the Freka-PEXACTSystem was 20 minutes (IQR: 15; 25 minutes) and for the pull technique was 15 minutes (IQR: 12; 20 minutes) $P<0.001$.

PEG placement using the pull technique was significantly faster in patients with $\mathrm{BMI}<19$ in comparison with the puncture technique $(P=0.09)$. No differences were found with regard to normal and overweight patients ( $>$ Fig. 2 ).

The difference in median duration of PEG placement between the pull and the puncture group was 10 minutes for stage T1 tumors $(P=0.002), 8$ minutes for T2, 7 minutes for T3 and 3 minutes for T4 tumors ( $\triangleright$ Fig. 3 ).

Tumor localization had no impact on procedure time $(P=$ 0.87 ). The Karnofsky Index and a post-surgery state did not have any influence on duration of PEG placement either. There was no statistically significant difference in propofol dosages between the pull and the puncture technique (not shown). 


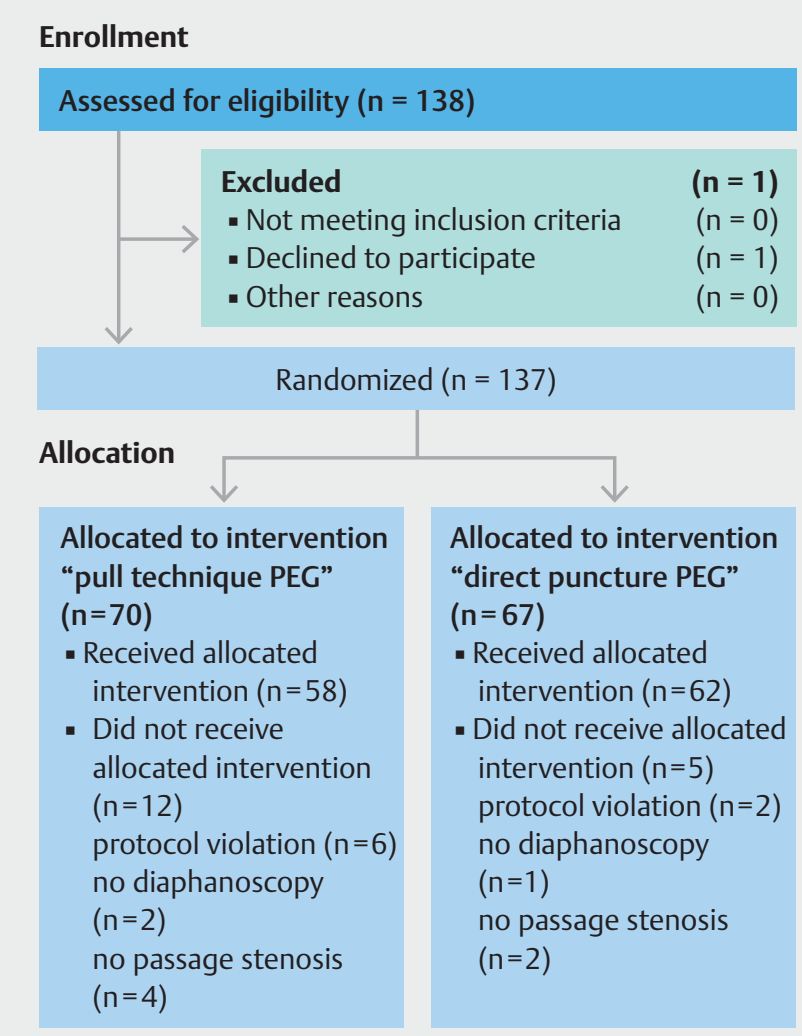

- Fig. 1 Patient randomization, allocation and per protocol intervention.

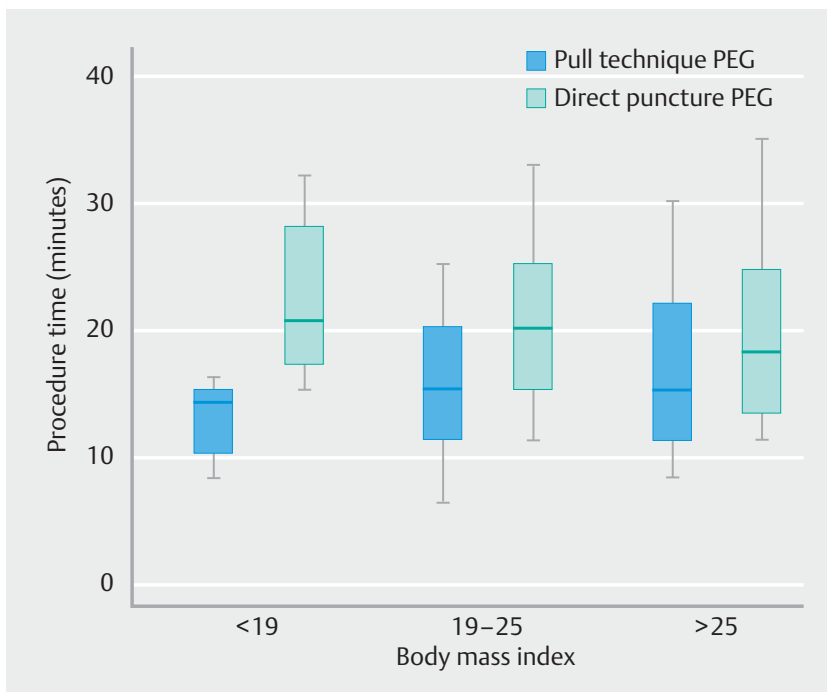

Fig. 2 Box blot analysis of BMI vs. PEG procedure time. In underweight patients, the pull technique was less time-consuming $(P=0.09)$.

\section{PEG adverse events}

Five patients suffered peri-interventional AEs. Two cases involved bleeding, which was treated endoscopically. Three patients suffered a peri-interventional decrease of oxygen satura-

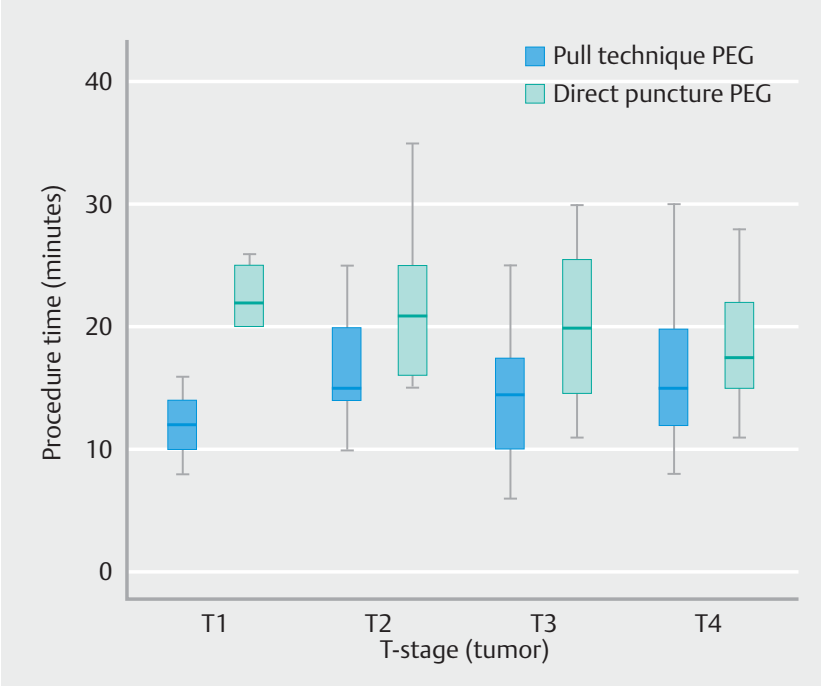

- Fig. 3 Box blot analysis of tumor T-stage vs. PEG procedure time. The pull technique was significantly faster only in $\mathrm{T} 1$ tumors $(P=0.002)$.

tion that necessitated increased oxygen supplementation, one of whom required supplementary intermittent mask-administered respiration. No serious AEs occurred.

Statistically significant differences emerged 1 month into the post-interventional period, especially with regard to appearance of rubor (pull $(n=4)$ vs. puncture $(n=18), P=0.004)$. Pain at the placement site was found only in the puncture group (pull $(n=0)$ vs. puncture $(n=5), P=0.059)$. Other criteria of inflammation, such as swelling or the occurrence of pus, did not differ significantly between both groups.

Overall, short-term AEs within 6 months occurred most frequently in the puncture group ( $n=33$ vs. $n=14$ in the pull group $P=0.0011$ or $P<0.003)$. Specific AEs are shown in $>$ Fig. 4 .

AEs did not emerge significantly more frequently in patients with cancer of the oral cavity, especially with stage T4 tumors or when the tumor was not resected prior to PEG placement. Patients who used the feeding tube did not experience AEs more frequently than non-users.

Three months after PEG insertion and later, AE were rare. Between 3 and 6 months after PEG insertion, 3 button PEGs (after Pexact removal) dislocated. The diameter of a button PEG (filled with $5 \mathrm{cc}$ sterile saline) was equal to the internal fixation disc of the pull technique PEG ( $25 \mathrm{~mm})$, as documented by the manufacturer. No relevant PEG-related AEs were noted later than 6 months after PEG insertion. There were no cases that showed any indication of metastasis formation at the gastrostomy fistula.

\section{Patient satisfaction}

Patient satisfaction with the percutaneous feeding tube was determined by means of a visual analogue scale (VAS). Within the first 3 weeks, patients without AEs were significantly more satisfied compared to patients with AEs according to their feedback on the Days 3, 7 and 21 of follow-up $(P=0.033, P=0.012$, 


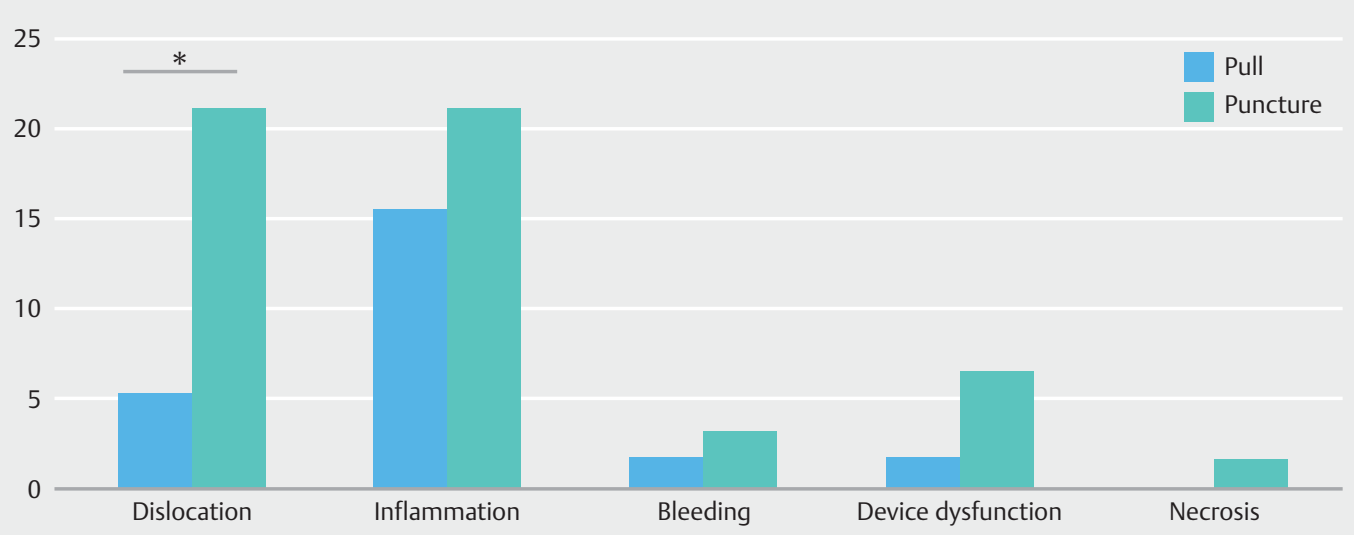

Fig. 4 Percentage of short-term adverse events for the pull vs. the puncture PEG technique within 6 months, ${ }^{*} P<0.005$, otherwise not significant.

$P=0.040$ respectively). We did not notice any significant differences between the pull and the puncture group.

\section{Long-term usage characteristics}

After 3 months, $78 \%$ of the study participants returned for medical examination; $6 \%$ died before their follow-ups could take place. After 6 months, the proportion of patients examined was $60 \%$, whereas the percentage of deceased patients rose to $10 \%$.

The PEG tube was used without any essential differences between the study groups during the 5-year follow-up period, with an overall usage, at least temporarily, by 101 of the 120 patients $(84.2 \%)$. Time between PEG insertion and death was no different between the pull and the puncture group $(P=$ 0.63; - Fig.5). Total enteral nutrition via the PEG was applied in 84 patients $(70 \%)$. A scheduled PEG removal after finishing multimodal tumor therapy was possible in 49 patients $(40.8 \%)$. No significant differences between the pull and the puncture group were found with regard to

- frequency of scheduled PEG change $(P=0.95)$

- time between last PEG usage and death ( $\triangleright$ Fig. 5) $(P=0.60)$

- PEG usage $(P=0.86)$

- total enteral nutrition via PEG $(P=0.079)$

Concerning increase in weight (BMI) and self-reliant care (Karnofsky Index), there were no relevant differences between the pull and the puncture group during follow-up.

\section{Discussion}

In this prospective, open, randomized study, we report for the first time on long-term PEG-related AEs and usage characteristics in a large cohort of patients with epithelial tumors of the upper gastrointestinal tract. Contrary to our hypothesis, we demonstrated that the innovative direct puncture device is associated with a higher rate of short-term PEG-related AEs in comparison with the traditional pull technique. This was not associated, however, with a reduced usage rate or impaired patient satisfaction.

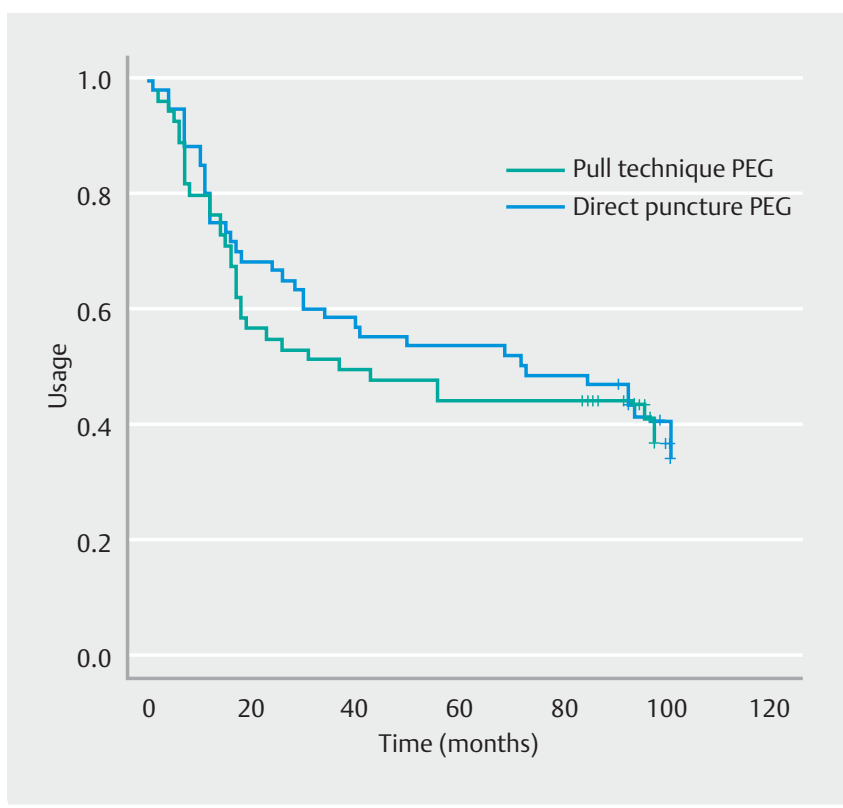

Fig. 5 The time between PEG insertion and death did not differ between the pull and the puncture groups $(P=0.63)$.

Our prospectively observed patient cohort experienced a low rate of inflammatory AEs (15.5\% and $21 \%$ in the pull and the puncture group, respectively). This is lower than reported in a recent retrospective study of 1625 patients (median follow-up 254 days) from 8 Korean hospitals, who received a pull technique PEG. In this study, $81.1 \%$ of all patients had a PEG inflammation [16]. This remarkable difference may be explained by the fact that our study used strict prophylactic antibiotic supplementation [17] and the PEG procedure was carried out in a specialized endoscopy unit using established standard operating procedures. We believe that our favorable results are not due to underreporting as all patients were carefully followed by experienced nutrition assistants and a standardized protocol was used. 
The direct puncture technique was associated with a longer procedure time. This is well in accord with a recently published retrospective comparative case series of 113 patients [18] and may simply be explained by the time-consuming performance of the 2 additional gastropexies aside the PEG. Our detailed subgroup analysis, however, revealed that the difference in procedure duration actually stems from the underweight and T1 tumor groups. The pull technique was faster in low-weight patients with sparse visceral fat and even small and less obstructing T1 tumors. Obstruction of the upper gastrointestinal tract is a relevant factor only for the pull technique as all tumors had to be passable by a standard gastroscope as per protocol. Consequently, the puncture technique was equally time-consuming in all T stages. Longer intervention times in the puncture group were not associated with higher propofol doses or peri-interventional adverse events. Therefore, this difference will not be present in most patients with tumors of the upper gastrointestinal tract and is of limited clinical relevance in lowweight and $\mathrm{T} 1$ tumor patients.

A secondary endpoint of our study was the comparison of tumor seeding at the PEG insertion site. Several authors have advocated the puncture technique on the grounds that it prevented implantation metastases. However, case reports show that PEG insertion using this technique does not eliminate the risk of direct tumor seeding [5, 6,19]. A possible explanation for the occurrence of tumor seeding could be that the gastroscope tip implants cells directly into the gastric wall during transillumination. It could also be the result of swallowed tumor cells settling into the fresh gastric wound, which would have caused the metastases to occur regardless of the method of PEG insertion. Fortunately, none of the patients in our study developed a PEG metastasis. Nevertheless, we support previous authors' advice to avoid passage of the gastroscope deep into the stomach. This may help to avoid direct contact with the gastric wall during transillumination to prevent inadvertent seeding of the primary tumor to the PEG wound [19].

During the follow-up period of 4.5 years (median), randomization to the pull or the puncture technique had no influence on the usage characteristics. About 5 of 6 patients who received a PEG used it over the long term without any differences in usage characteristics between the 2 devices used in this study. A scheduled PEG removal after finishing multimodal tumor therapy was possible in more than $40 \%$ of our patients. With regard to quality of life, the puncture technique could have an advantage over the pull technique as it does not require another endoscopy for PEG removal. However, that question has not been addressed in this trial.

The Freka PEG, which was used for the pull technique, was manufactured with polyurethane, whereas the Freka-PEXACT was manufactured with synthetic silicone rubber. Both materials are highly bio- and tissue compatible. Theoretically, there is slightly more irritation with silicone-based tubes because of the roughness of the surface, but in clinical practice, insufficient sealing due to incorrect use or maintenance errors may be superimposed on this factor. Differences between the materials, therefore, may be more reflective of optical or functional aspects (color changes, occlusion etc.) than relevant to AEs. A mi- nor bias especially for the endpoint "rubor," however, could be conceivable [20,21].

We have not assessed glucose tolerance status in our patients. That may be a weakness of our protocol as uncontrolled diabetes is a risk factor for wound infection. Albumin was not measured systematically and there only BMI can be used as an objective measure of nutrition. Howeve $4 r$, BMI had no influence on any PEG complications.

\section{Conclusion}

In conclusion, our results favor the pull technique for application of a PEG in patients with endoscopically passable tumors of the upper gastrointestinal tract due to fewer short-term AEs. Both the pull and the puncture system contributed equally to secure long-term use and patient satisfaction. The individual choice of an appropriate technique should be based on the patient's informed consent, individual factors such as BMI or tumor size, and the operator's experience and the facilities available.

\section{Acknowledgements}

This trial has not been registered through any of the registries approved by the International Committee of Medical Journal Editors as this was not mandatory at the time of initiation of our study in 2007. This study was supported in part by a grant from Fresenius Kabi AG, Bad Homburg, Germany. The sponsor did not have any influence on the trial concept, protocol, data collection, data analysis and manuscript preparation.

\section{Competing interests}

None

References

[1] Datema FR, Ferrier MB, Baatenburg de Jong RJ. Impact of severe malnutrition on short-term mortality and overall survival in head and neck cancer. Oral Oncol 2011; 47: 910 - 914

[2] Arends J, Bodoky G, Bozzetti F et al. ESPEN Guidelines on Enteral Nutrition: Non-surgical oncology. Clin Nutr 2006; 25: 245 - 259

[3] Gauderer MW, Ponsky JL, Izant RJ Jr. Gastrostomy without laparotomy: a percutaneous endoscopic technique. J Pediatr Surg 1980; 15: 872 875

[4] Preyer S, Thul P. Gastric metastasis of squamous cell carcinoma of the head and neck after percutaneous endoscopic gastrostomy: report of a case. Endoscopy 1989; 21: 295

[5] Cappell MS. Risk factors and risk reduction of malignant seeding of the percutaneous endoscopic gastrostomy track from pharyngoesophageal malignancy: a review of all 44 known reported cases. Am J Gastroenterol 2007; 102: 1307-1311

[6] Ananth S, Amin M. Implantation of oral squamous cell carcinoma at the site of a percutaneous endoscopic gastrostomy: a case report. $\mathrm{Br}$ ] Oral Maxillofac Surg 2002; 40: 125-130 
[7] Russell TR, Brotman M, Norris F. Percutaneous gastrostomy. A new simplified and cost-effective technique. Am J Surg 1984; 148: 132 137

[8] Tucker AT, Gourin CG, Ghegan MD et al. 'Push' versus 'pull' percutaneous endoscopic gastrostomy tube placement in patients with advanced head and neck cancer. Laryngoscope 2003; 113: 1898-1902

[9] Maetani I, Tada T, Ukita T et al. PEG with introducer or pull method: a prospective randomized comparison. Gastrointest Endosc 2003; 57 : $837-841$

[10] Maetani I, Yasuda M, Seike M et al. Efficacy of an overtube for reducing the risk of peristomal infection after PEG placement: a prospective, randomized comparison study. Gastrointest Endosc 2005; 61: $522-527$

[11] Toyama Y, Usuba T, Son K et al. Successful new method of extracorporeal percutaneous endoscopic gastrostomy (E-PEG). Surg Endosc 2007; 21: $2034-2038$

[12] Horiuchi A, Nakayama $\mathrm{Y}$, Tanaka $\mathrm{N}$ et al. Prospective randomized trial comparing the direct method using a $24 \mathrm{Fr}$ bumper-button-type device with the pull method for percutaneous endoscopic gastrostomy. Endoscopy 2008; 40: $722-726$

[13] Hiki N, Maetani I, Suzuki Y et al. Reduced risk of peristomal infection of direct percutaneous endoscopic gastrostomy in cancer patients: comparison with the pull percutaneous endoscopic gastrostomy procedure. J Am Coll Surg 2008; 207: 737 - 744

[14] Van Dyck E, Macken EJ, Roth B et al. Safety of pull-type and introducer percutaneous endoscopic gastrostomy tubes in oncology patients: a retrospective analysis. BMC Gastroenterol 2011; 11: 23
[15] Lim JH, Choi SH, Lee C et al. Thirty-day mortality after percutaneous gastrostomy by endoscopic versus radiologic placement: a systematic review and meta-analysis. Intest Res 2016; 14: 333 - 342

[16] Lee C, Im JP, Kim JW et al. Risk factors for adverse events and mortality of percutaneous endoscopic gastrostomy: a multicenter, retrospective study. Surg Endosc 2013; 27: 3806 - 3815

[17] Ahmad I, Mouncher A, Abdoolah A et al. Antibiotic prophylaxis for percutaneous endoscopic gastrostomy - a prospective, randomised, double-blind trial. Aliment Pharmacol Ther 2003; 18: $209-215$

[18] Ohno T, Ogawa A, Yanai M et al. The usefulness and safety of the introducer technique using a bumper-button-type device as compared with the pull method for percutaneous endoscopic gastrostomy. Surg Laparosc Endosc Percutan Tech 2015; 25: e1 - 4

[19] Teh JL, Wong RK, Gowans M et al. Gastric metastases of oral carcinoma resulting from percutaneous endoscopic gastrostomy placement via the introducer technique. Gastroenterol Rep (Oxf) 2013; 1: 211 213

[20] Sartori S, Trevisani L, Nielsen I et al. Longevity of silicone and polyurethane catheters in long-term enteral feeding via percutaneous endoscopic gastrostomy. Aliment Pharmacol Ther 2003; 17: 853 856

[21] Blacka J, Donoghue J, Sutherland $M$ et al. Dwell time and functional failure in percutaneous endoscopic gastrostomy tubes: a prospective randomized-controlled comparison between silicon polymer and polyurethane percutaneous endoscopic gastrostomy tubes. Aliment Pharmacol Ther 2004; 20: 875-882 